\title{
Bi-level Optimization Application for Urban Traffic Management
}

\author{
Krasimira Stoilova \\ Institute of Information and Communication \\ Technologies - Bulgarian Academy of Sciences, \\ Acad. G. Bonchev str. bl.2, 1113 Sofia, Bulgaria \\ Email: k.stoilova@hsi.iccs.bas.bg
}

\author{
Todor Stoilov \\ Institute of Information and Communication \\ Technologies - Bulgarian Academy of Sciences, \\ Acad. G. Bonchev str. bl.2, 1113 Sofia, Bulgaria \\ Email: todor@hsi.iccs.bas.bg
}

\begin{abstract}
A bi-level modeling for traffic lights optimization is presented in the paper. The bi-level modeling allows increasing the set of control influences, the number of constraints and applies two goal functions in hierarchical order. The bi-level formalism allows integration of small optimization problems in hierarchical order to a complex interconnected and complicated optimization problem. These features have been applied for optimal control of traffic lights in urban network. The bilevel problem formulation allows to minimize the queue lengths of vehicles and to maximize the outgoing flows from arterial directions. Both control influences of the green light durations and time cycles are evaluated as optimal bi-level control influences.
\end{abstract}

\section{INTRODUCTION}

$\mathrm{T}$ RAFFIC congestion in urban networks is an everyday problem, which has negative influences on many human and society activities. The traffic congestions result in queuing of vehicles in urban crossroad sections and generate excessive delays, increasing pollution, degradation of infrastructure. The congestion events can be changed by modifying the urban infrastructure. But it is easy to conclude that such "off line" management of a transportation system will not give reasonable results in time.

That is why from control point of view the management of the traffic behavior with on-line strategies, adapting the control influences to dynamical urban behavior is the most appropriate solution. The application of traffic lights control at intersections is power solution to reduce the bottlenecks on the network. Such traffic lights control is a competitive strategy, which improves the traffic mobility [1]. This optimization allows decreasing the travel time, reducing the fuel consumptions, traffic emissions, and noise. The optimization of the traffic lights duration is regarded as power tool for regulation the traffic flows at the intersections.

The control influences on the traffic conditions are not so many: duration of the green lights and/or the split of the cycle; the cycle duration, which concerns the time for all lights including green, red and amber one; the phase between the traffic lights on sequential road intersections. These three types of control influences must be evaluated as solutions of

This work is supported by Project KP06-H37/6 "Modelling and optimization of urban traffic in network of crossroads" with the Bulgarian Research Fund. appropriate control problem, which considers the current traffic state on urban intersections. The evaluation of the values of these control parameters can resolve problems like:

- Traffic congestion: reducing oversaturation at intersections;

- Traffic fluctuations: change of the current plan of the traffic lights to respond to considerable change of traffic intensity;

- Change of urban infrastructure due to road accident, closed street for human events, redirection of heavy vehicles.

A control problem, which optimizes only one isolated intersection cannot avoid the generation of bottlenecks. The congestions do not allow the transport to use the urban capacity because the overall traffic system degrades and the car motion is restricted on the overall urban network. Hence, the traffic light control has to be implemented on a network infrastructure and respectively to be applied control influences both with traffic lights, cycle durations and phase/time delays between the green lights achieving 'green waves' on important directions

Obviously, the definition of such complex optimization problem, where the transportation network comprises many intersections will generate high dimensions of the control problem and computational requirements will arise for real time solutions. Additionally, the increase of the control space will make the control problem also non-linear and hard for analytical definition.

The approach which is applied in this research is the integration and interconnection of small optimization problems in hierarchical order by means to define and to solve more complex control problem for traffic optimization. The small optimization problem has only one type of control influence: traffic lights green light duration or cycle duration. But making integration of these problems in hierarchical order the complicated one will have extended control space containing both types of control influences. This research targets the development of optimal control strategy, which implements simultaneously in optimal manner both types of control influences: the duration of the 
green lights and the duration of the traffic lights cycle. Such control problem has power to change the traffic behavior with extended set of control parameters, which is a benefit for these control processes. Such increase of the control space in this research is achieved by integration of control problem in a bi-level hierarchical procedure. The bi-level approach is implemented for the definition of a complex optimization problem $[4,18]$. The problem is applied for the traffic optimization on arterial urban network in town of Sofia. The numerical simulations give advantages to the bilevel optimal control of the traffic lights and cycles in the urban transportation network in Sofia. A self-adaptive traffic signal control system adjusting the signal timing parameters in real time is considered.

\section{OPTIMAL CONTROL OF THE TRAFFIC LIGHTS ON INTERSECTION}

The design of the traffic lights stages at isolated intersection is standardized in industrial countries [2], [3]. For analytical overview about traffic signal control one can refer to [4]. The current practice of the application of the traffic engineering and traffic signals is presented in [5], [6]. A review of traffic control strategies, which are applied frequently in traffic systems are analyzed in [7], [8]. The optimization of the traffic signals under specific requirements is considered in [9]. A self-adaptive traffic control system, adjusting the signal timing parameters in real time is considered in [10]. This paper does not present formal definition of the optimization problem. Only the traffic lights are evaluated on procedural way. The cycle of timing is not considered as optimal problem solution.

Model predictive approach is also used in urban traffic management [11]. Store-and-forward modeling is applied and minimization of queue lengths in front of the intersection is performed. The duration of the green lights is estimated and controlled by simulation environment. The cycle duration and offset are not taken into consideration.

The store-and-forward modeling was applied for definition of optimal control problem in [12]. The solution of the problem gives plans only for the traffic lights.

Requirements towards public transport by optimization of traffic signals are considered in [13], [14]. Optimal signal settings are calculated only for the traffic lights. Simulation tools are also used for optimization of traffic lights [15].

Meta heuristics algorithms are also applied for intersection control [16].

All these methods try to solve optimization problem with objective value the traffic lights cycle duration or the green light duration that represent one-criterion optimization problem. But the formalism in optimization theory moves to the definition of more complex problems, based on hierarchical system theory. Hierarchically interconnected optimization problems allow to be extended the space of the control influences and parameters. Because the solution of such problems is quite complex, mainly the bi-level formalism is used for transportation problems.

\section{APPLICATION OF BI-LEVEL OPTIMIZATION IN TRANSPORTATION}

The bi-level approach is based on hierarchical integration of both optimization problems. The bi-level problems appeared firstly in game theory concerning the behavior and negotiations between leader and follower. Up to date bibliographical reviews about the bi-level and multilevel programming one can find in [17], [18]. The formal definition of a bi-level optimization problem is

$$
\begin{gathered}
\min _{y} F(x, y) \\
x \equiv \arg \left\{\begin{array}{l}
\min _{x} f(x, y) \\
g(x, y) \leq 0
\end{array}\right.
\end{gathered}
$$

$x \in X, y \in Y, F, f$-scalar functions, $g$ - set of constraints.

The solution of bi-level optimization is not an easy task, however it exists a tendency for its intensive application in different transportation problems.

In [19] goods must be transported and distributed from $m$ sources to $n$ destinations. But the transportation is divided hierarchically on several layers, which makes different priority to each destination. The bi-level objectives insist minimization of shipment time at each hierarchical level.

In [20] is defined a logistic bi-level problem. It concerns the distribution of a set of logistics centers and customer transportation costs. The lower optimization problem minimizes the customer costs together with satisfying their demands. The upper problem minimizes the cost of establishing the logistics centers.

A bi-level formalism is also used in public transportation [21]. It has been optimized the time interval between buses, considering the capacity of each bus. The user choice of routes is kept as low level problem. The bi-level transportation problem on upper level minimizes the travel costs, and on the lower level bus transportation scheme is considered [22].

Application of bi-level optimization in the domain of transportation policies one can find also in [23]; for network transportation - in [24]; for special kind of goods and their transportation in [25], [26]; for intermodal transport - [27], [28] ; for locating of logistics in [29]. Timing considerations in transportation are addressed in [4], [7], [30], [31].

This analysis illustrates that the bi-level optimization formalism has entered in the transportation domain, giving benefits for the design, control, and decision making problems. The power of the bi-level formalism is based on the opportunity the optimization problem to accommodate more constraints, to extend the space of the optimization parameters, to apply in hierarchical order two goal functions. Short explanations of these features are given with the comparison between the bi-level problem (1) and the classical optimization problem (2):

$$
\begin{aligned}
& \min _{x} f(x) \\
& g(x) \leq 0
\end{aligned}
$$

The classical optimization problem (2) evaluates as optimal solutions the set of parameters $\mathbf{x}$. The set of optimal solutions in the bi-level problem (1) is higher in comparison with (2) because (1) contains both sets $\mathbf{x}$ and $\mathbf{y}$. The classical 
problem (2) takes in consideration small set of constraints, $g(x)$, while the bi-level problem considers bigger set of requirements, simultaneously $G(x, y)$ and $g(x, y)$. Finally, the bi-level problem keeps two criteria on their max/min values, $F(x, y)$ and $f(x, y)$, in comparison with the single criteria $f(x)$ for problem (2). Hence, the bi-level problem has bigger potential to consider more requirements in the optimization problem. This is one of the reasons the bi-level formalism to be applied intensively in different domains for optimization of resource allocation, control policies, design processes. That is why the described advantages are applied in this research for integration of optimization problems for increase of the control space of a transportation problem both with the traffic lights duration and the traffic light cycle.

\section{TRANSPORT NETWORK UNDER BI-LEVEL OPTIMIZATION}

The urban network, which is considered, is one of the main arterial streets of Sofia town. This urban place very often generates transportation bottlenecks. Free cars, public transportation and pedestrian flows take place in this urban network. On close distances, five sequential intersections are controlled with traffic lights. After assessment of the behavior of the traffic flows, the intensities of inputs/outputs of vehicles, it has been identified the traffic light plans on this network, which currently keep constant parameters for the green light durations and cycle time. The goal of this research leads to evaluation of new values of the traffic lights parameters: green phases and cycle duration.

The topology of the transport network is presented in Figure 1. The main inputs of vehicles come from left and right direction of this arterial network. The crossing intersections reduce the main traffic direction and generate congestions inside of the arterial network. Such congestions make degradation for the main transport directions (left to right and opposite) and also increase the waiting vehicles, which cross the main street. Five regulated with traffic lights intersections have to support such plans for the green lights and cycle durations by means the queues and waiting vehicles into the arterial street to be prevented from congestions.

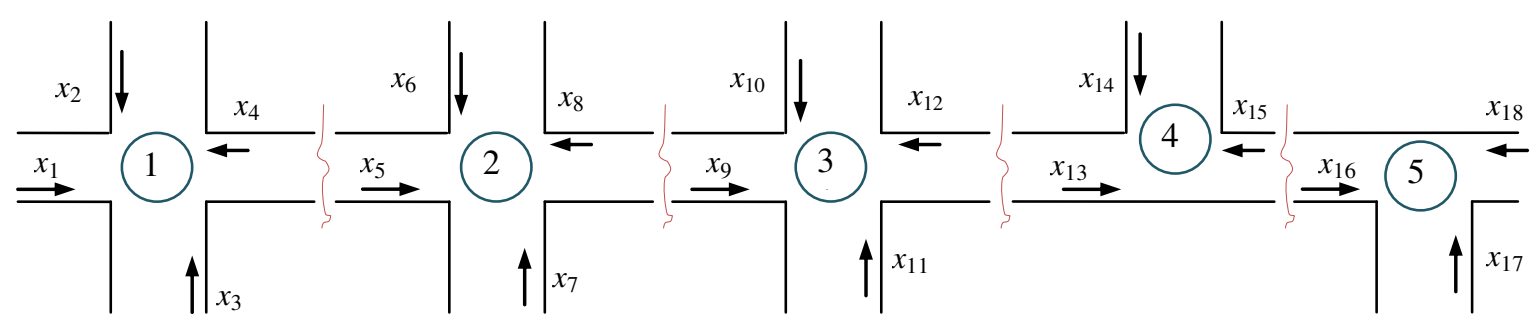

Fig.1 Transportation network under bi-level control

The bi-level optimization goal is to minimize the queue lengths in front of the crossroad sections and to keep such volumes of cars, which will not generate bottlenecks on the arterial direction of the network from left to right and opposite.

\section{V.Definition of the Bi-LeVEl OPTIMIZATION PROBLEM}

The bi-level optimization problem is defined as integration if two optimization sub-problems, Fig.2. The low level sub-problem targets the minimization of the queue lengths $\mathbf{x}$ in front of the traffic lights of the intersections. This minimization is done by calculating the duration of the green lights $\mathbf{u}$ for all intersections. The lower level problem applies as predefined parameters the duration of the cycle durations c. The last are evaluated by the upper level optimization sub-problem. The upper-level optimization sub-problem takes as predefined parameters the queue lengths $\mathbf{x}$ and the green duration $\mathbf{u}$ and it evaluates optimal cycles $\mathbf{c}$ for maximization of the outflows of the arterial directions. Hence, the bi-level problem is constituted as two interconnected sub-problems.

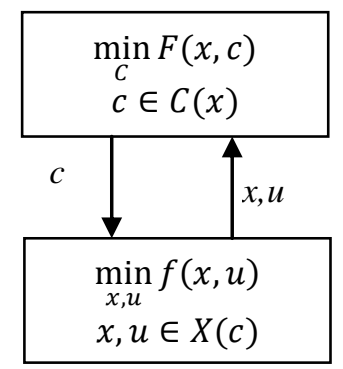

Fig.2. Hierarchical optimization of two optimization sub-problems

The solutions of the bi-level problem will give optimal values of the queue lengths, green light durations and the cycle durations for the traffic lights.

\subsection{Definition of the low level sub-problem}

The lower level problem aims to minimize the queue lengths. The common formulation of this problem is

$$
\begin{aligned}
& \min _{x} f(x, u) \\
& x, u \in X(c)
\end{aligned}
$$


The goal function is chosen in a quadratic form

$$
\min \left(x^{2}+u^{2}\right) \text {. }
$$

The analytical description of the queue lengths of subproblem (3)-(4) is based on the store and forward modeling. The general form of the store and forward modeling is given by relation (6)

$$
\begin{aligned}
& \mathbf{x}(\mathbf{k}+\mathbf{1})=\mathbf{x}(\mathbf{k})+\mathbf{x}_{\text {in }}-\mathbf{x}_{\text {out }}, \\
& \mathbf{x}_{\text {out }}=\mathbf{s}^{(\mathbf{i})} \mathbf{u}+\overline{\boldsymbol{s}}^{(\mathbf{i})} \mathbf{u},
\end{aligned}
$$

where $k+1$ is the current control cycle, $\mathbf{x}$ are the queue lengths in the previous $k$ and the current $k+1$ control cycle; $\mathbf{x}_{\text {in }}$ are the inflows of vehicles at each intersection, $\mathbf{x}_{\text {out }}$ are the outgoing flows. The volume of outgoing vehicles $\mathbf{x}_{\text {out }}$ is managed by the duration of the green light of this intersection for direction $i$, where $\mathbf{s}^{(\mathbf{i})}$ is the saturation flow on this direction, $\overline{\boldsymbol{s}}^{(\mathbf{i})}$ is the saturation for the turning flows and $\mathbf{u}$ is the duration of the green light of the intersection.

We have to minimize all the queues in the network having in mind the store and forward model (6). The available traffic flows of the first crossroad section are graphically presented in Fig.3.

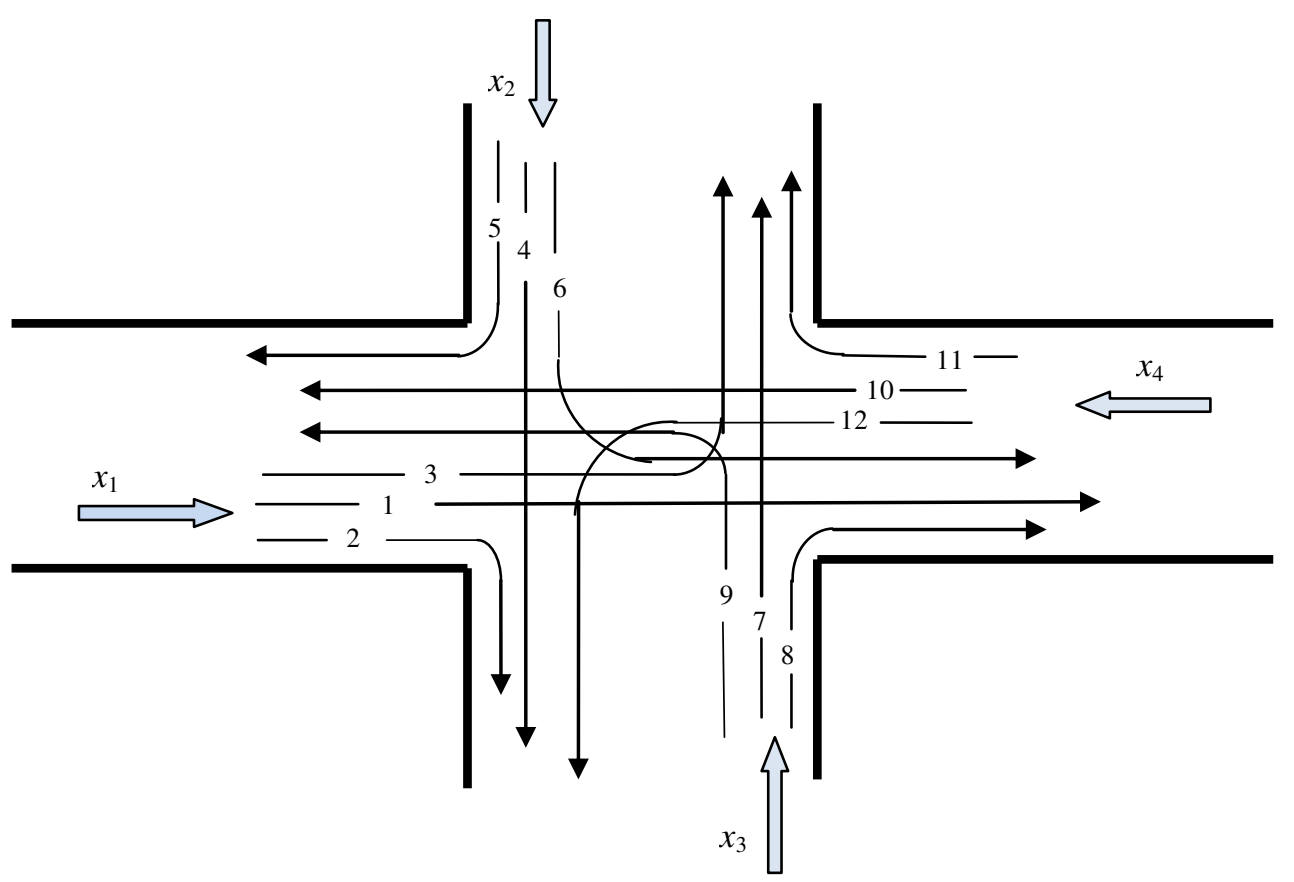

Fig.3. Traffic flows of the first crossroad section

For the first crossroad section (node) the duration of the green light in horizontal direction is noted as $u_{1}$ and in vertical direction $u_{2}$. The flow saturations in horizontal and vertical directions are $s_{1}$ and $s_{2}$. We suppose that the right curve has saturation $\overline{s_{1}}$ in horizontal and $\overline{S_{2}}$ in vertical direction. The levels of saturations for the left curves are respectively $\frac{1}{2} \overline{s_{1}}$ and $\frac{1}{2} \overline{s_{2}}$.

These levels of the saturation flows are defined according to the infrastructural dimensions. Following Fig.3, the first crossroad section manages twelve traffic flows, noted sequentially from 1 to 12 with corresponding outflows, computed according to the duration of the green lights $\mathbf{u}$ and the corresponding saturation flows in direction:

$$
\begin{aligned}
& \text { "1" } \rightarrow u_{1} s_{1} ; \quad \text { "2" } \rightarrow u_{1} \overline{s_{1}} ; \quad \text { " } 3 \text { " } \rightarrow \frac{1}{2} u_{1} \overline{s_{1}} \text {; } \\
& \text { "4" } \rightarrow u_{2} s_{2} ; \text { " } 5 \text { " } \rightarrow u_{2} \overline{s_{2}} ; \quad \text { "6" } \rightarrow \frac{1}{2} u_{2} \overline{s_{2}} \text {; } \\
& \text { "7" } \rightarrow u_{2} s_{2} ; \text { " } 8 \text { " } \rightarrow u_{2} \overline{s_{2}} ; \quad \text { "9" } \rightarrow \frac{1}{2} u_{2} \overline{s_{2}} \text {; } \\
& \text { "10" } \rightarrow u_{1} s_{1} ; " \text { " } 11 " \rightarrow u_{1} \overline{s_{1}} ; \quad \text { " } 12 " \rightarrow \frac{1}{2} u_{1} \overline{s_{1}} \text {. }
\end{aligned}
$$

The same form of modelling is applied for the second crossroad section (node), where the green light duration in horizontal and vertical directions are noted respectively $u_{3}$ and $u_{4}$ and the horizontal and vertical levels of saturation flows are $s_{3}$ and $s_{4}$. The set of outflows are defined as:

$$
\begin{aligned}
& \text { "13" } \rightarrow u_{3} s_{3} ; " 14 " \rightarrow u_{3} \overline{S_{3}} ; \quad \text { " } 15 " \rightarrow \frac{1}{2} u_{3} \overline{s_{3}} \text {; } \\
& \text { "16" } \rightarrow u_{4} s_{4} ; \quad \text { "17" } \rightarrow u_{4} \overline{s_{4}} ; \quad \text { "18" } \rightarrow \frac{1}{2} u_{4} \overline{s_{4}} \text {; } \\
& \text { "19" } \rightarrow u_{4} s_{4} ; \quad \text { "20" } \rightarrow u_{4} \overline{s_{4}} ; \quad \text { " } 21 \text { " } \rightarrow \frac{1}{2} u_{4} \overline{s_{4}} \text {; } \\
& \text { " } 22 \text { " } \rightarrow u_{3} s_{3} ; " 23 \text { " } \rightarrow u_{3} \overline{s_{3}} ; \quad \text { " } 24 \text { " } \rightarrow \frac{1}{2} u_{3} \overline{s_{3}} \text {. }
\end{aligned}
$$

For the third crossroad section (node) the corresponding parameters are $u_{5}$ and $u_{6}$ for the green phase durations and $s_{5}$ and $s_{6}$ for the saturation flows. The traffic outflows are noted as:

$$
\begin{aligned}
& \text { " } 25 \text { " } \rightarrow u_{5} s_{5} ; \quad \text { " } 26 " \rightarrow u_{5} \overline{s_{5}} ; \quad \text { " } 27 \text { " } \rightarrow \frac{1}{2} u_{5} \overline{s_{5}} \text {; } \\
& \text { " } 28 \text { " } \rightarrow u_{6} s_{6} ; \text { " } 29 \text { " } \rightarrow u_{6} \overline{s_{6}} ; \quad \text { "30" } \rightarrow \frac{1}{2} u_{6} \overline{s_{6}} \text {; } \\
& \text { " } 31 \text { " } \rightarrow u_{6} s_{6} ; " 32 " \rightarrow u_{6} \overline{s_{6}} ; \quad \text { " } 33 \text { " } \rightarrow \frac{1}{2} u_{6} \overline{s_{6}} \text {; } \\
& \text { " } 34 \text { " } \rightarrow u_{5} s_{5} ; " 35 " \rightarrow u_{5} \overline{s_{5}} ; \quad \text { " } 36 \text { " } \rightarrow \frac{1}{2} u_{5} \overline{s_{5}} \text {. }
\end{aligned}
$$


For the fourth node the notation used are $\left(u_{7}\right.$ and $u_{8}, s_{7}$ and $\overline{S_{8}}$ ) and the outgoing traffic flows are:

$$
\begin{array}{ll}
\text { "37" } \rightarrow u_{7} s_{7} ; & \text { "38" } \rightarrow \frac{1}{2} u_{7} \overline{s_{7}} ; \\
" 39 " \rightarrow u_{8} \overline{s_{8}} ; & " 40 " \rightarrow \frac{1}{2} u_{8} \overline{s_{8}} ; \\
" 41 " \rightarrow u_{7} s_{7} ; & " 42 " \rightarrow u_{7} \overline{s_{7} .}
\end{array}
$$

The last fifth node applies notations $\left(u_{9}\right.$ and $u_{10}, s_{9}$ and $\left.s_{10}\right)$ with outgoing traffic flows :

$$
\begin{aligned}
& \text { "43" } \rightarrow u_{9} s_{9} ; \quad \text { " } 44 \text { " } \rightarrow u_{9} \overline{S_{9}} \text {; } \\
& \text { “ } 45 \text { " } \rightarrow u_{10} \overline{s_{10}} ; \quad \text { " } 46 \text { " } \rightarrow \frac{1}{2} u_{10} \overline{S_{10}} \text {; } \\
& \text { " } 47 \text { " } \rightarrow u_{9} S_{9} ; \quad \text { " } 48 \text { " } \rightarrow \frac{1}{2} u_{9} \overline{S_{9}} \text {. }
\end{aligned}
$$

The level of the queue lengths $(x)$ in front of the traffic lights is changed for each traffic light control cycle. Applying the store-and-forward model the levels of the queue lengths for the current control cycle depend from the residual queues from the previous control cycle $\left(x_{i \mathrm{o}}\right), i=1, \ldots, 48$, increased by the incoming flows $\left(x_{i n}\right)$, decreased by the outgoing flows on each direction (straight ahead, right and left curves). Applying the relations of the store-and forward modeling for the urban network from Fig.1, the relations of the queues according to (6) define a set of 18 inequalities, requiring control policy, which results in less queue lengths for the current control cycle:

$$
\begin{aligned}
& x_{1} \leq x_{1 o}+x_{1 \text { in }}-u_{1} s_{1}-u_{1} \overline{s_{1}}-\frac{1}{2} u_{1} \overline{s_{1}} \\
& x_{2} \leq x_{2 o}+x_{2 \text { in }}-u_{2} s_{2}-1.5 u_{2} \overline{s_{2}} \\
& x_{3} \leq x_{3 o}+x_{3 i n}-u_{2} s_{2}-1.5 u_{2} \overline{s_{2}} \\
& x_{4} \leq x_{4 o}+u_{3} s_{3}+1.5 u_{4} \overline{s_{4}}-u_{1} s_{1}-1.5 u_{1} \overline{s_{1}} \\
& x_{5} \leq x_{5 o}+u_{1} s_{1}+1.5 u_{2} \overline{s_{2}}-u_{3} s_{3}-1.5 u_{4} \overline{s_{4}} \\
& x_{6} \leq x_{6 o}+x_{6 i n}-u_{4} s_{4}-1.5 u_{4} \overline{s_{4}} \\
& x_{7} \leq x_{7 o}+x_{7 i n}-u_{4} s_{4}-1.5 u_{4} \overline{s_{4}} \\
& x_{8} \leq x_{8 o}+u_{5} s_{5}+1.5 u_{6} \overline{s_{6}}-u_{3} s_{3}-1.5 u_{3} \overline{s_{3}} \\
& x_{9} \leq x_{9 o}+u_{3} s_{3}+1.5 u_{4} \overline{s_{4}}-u_{5} s_{5}-1.5 u_{5} \overline{s_{5}} \\
& x_{10} \leq x_{10 o}+x_{10 i n}-u_{6} s_{6}-1.5 u_{6} \overline{s_{6}} \\
& x_{11} \leq x_{11 o}+x_{11 \text { in }}-u_{6} s_{6}-1.5 u_{6} \overline{s_{6}} \\
& x_{12} \leq x_{12 o}+u_{7} s_{7}+u_{8} \overline{s_{8}}-u_{5} s_{5}-1.5 u_{5} \overline{s_{5}} \\
& x_{13} \leq x_{13 o}+u_{5} s_{5}+1.5 u_{6} \overline{s_{6}}-u_{7} s_{7}-0.5 u_{7} \overline{s_{7}} \\
& x_{14} \leq x_{14 o}+x_{14 i n}-1.5 u_{8} \overline{s_{8}} \\
& x_{15} \leq x_{15 o}+u_{9} s_{9}+0.5 u_{10} \overline{s_{10}}-u_{7} s_{7}-u_{7} \overline{s_{7}} \\
& x_{16} \leq x_{16 o}+u_{7} s_{7}+0.5 u_{8} \overline{s_{8}}-u_{9} s_{9}-u_{9} \overline{s_{9}} \\
& x_{17} \leq x_{17 o}+x_{17 \text { in }}-1.5 u_{10} \overline{s_{10}} \\
& x_{18} \leq x_{18 o}+x_{18 \text { in }}-u_{9} s_{9}+0.5 u_{9} \overline{s_{9}}
\end{aligned}
$$

In matrix form, the set of inequalities (7) is written as

$$
\mathbf{A}_{1} \mathbf{x}+\mathbf{A}_{\mathbf{2}} \mathbf{u} \leq \mathrm{B}
$$

The lower level sub-problem is defined in the form

$$
\begin{aligned}
& \min \left(\mathbf{x}^{\mathbf{T}} \mathbf{x}+\mathbf{u}^{\mathbf{T}} \mathbf{u}\right) \\
& \mathbf{A}_{\mathbf{1}} \mathbf{x}+\mathbf{A}_{\mathbf{2}} \mathbf{u} \leq \mathbf{B},
\end{aligned}
$$

where minimization of the queues $\mathbf{x}$ is considered in the goal function, $\mathbf{A}_{1}, \mathbf{A}_{2}, \mathbf{B}$ are corresponding matrices, derived from (7).
For the topology of the transportation network of Figure 1 , the relations (7) presented in form (8) give the set of constraints for the low level sub-problem as

$$
\begin{aligned}
& x_{1}+\left(s_{1}+1.5 \overline{s_{1}}\right) u_{1} \leq x_{1 o}+x_{1 i n} \\
& x_{2}+\left(s_{2}+1.5 \overline{s_{2}}\right) u_{2} \leq x_{2 o}+x_{2 i n} \\
& x_{3}+\left(s_{2}+1.5 \overline{s_{2}}\right) u_{2} \leq x_{3 o}+x_{3 i n} \\
& x_{4}+\left(s_{1}+1.5 \overline{s_{1}}\right) u_{1}-s_{3} u_{3}-1.5 \overline{s_{4}} u_{4} \leq x_{4 o} \\
& x_{5}-s_{1} u_{1}-1.5 \overline{s_{2}} u_{2}+s_{3} u_{3}+1.5 \overline{s_{4}} u_{4} \leq x_{5 o} \\
& x_{6}+\left(s_{4}+1.5 \overline{s_{4}}\right) u_{4} \leq x_{6 o}+x_{6 i n} \\
& x_{7}+\left(s_{4}+1.5 \overline{s_{4}}\right) u_{4} \leq x_{7 o}+x_{7 i n} \\
& x_{8}+\left(s_{3}+1.5 \overline{s_{3}}\right) u_{3}-s_{5} u_{5}-1.5 \overline{s_{6}} u_{6} \leq x_{8 o} \\
& x_{9}-u_{3} s_{3}-1.5 \overline{s_{4}} u_{4}+\left(s_{5}+1.5 \overline{s_{5}}\right) u_{5} \leq x_{9 o} \\
& x_{10}+\left(s_{6}+1.5 \overline{s_{6}}\right) u_{6} \leq x_{10 o}+x_{10 i n} \\
& x_{11}+\left(s_{6}+1.5 \overline{s_{6}}\right) u_{6} \leq x_{11 o}+x_{11 i n} \\
& x_{12}+\left(s_{5}+1.5 \overline{s_{5}}\right) u_{5}-s_{7} u_{7}-\overline{s_{8}} u_{8} \leq x_{12 o} \\
& x_{13}-s_{5} u_{5}-1.5 \overline{s_{6}} u_{6}+\left(s_{7}+0.5 \overline{s_{7}}\right) u_{7} \leq x_{13 o} \\
& x_{14}+1.5 \overline{s_{8}} u_{8} \leq x_{14 o}+x_{14 i n} \\
& x_{15}+\left(s_{7}-\overline{s_{7}}\right) u_{7}-s_{9} u_{9}-0.5 \overline{s_{10}} u_{10} \leq x_{15 o} \\
& x_{16}-s_{7} u_{7}-0.5 \overline{s_{8}} u_{8}+\left(s_{9}+\overline{s_{9}}\right) u_{9} \leq x_{16 o} \\
& x_{17}+1.5 \overline{s_{10}} u_{10} \leq x_{17 o}+x_{17 i n} \\
& x_{18}+\left(s_{9}+0.5 \overline{s_{9}}\right) u_{9} \leq x_{18 o}+x_{18 i n} \\
& u_{1}+u_{2}=\alpha_{1} C_{1} \\
& u_{3}+u_{4}=\alpha_{2} C_{2} \\
& u_{5}+u_{6}=\alpha_{3} C_{3} \\
& u_{7}+u_{8}=\alpha_{4} C_{4} \\
& u_{9}+u_{10}=\alpha_{5} \overline{C_{5}}
\end{aligned}
$$

\subsection{Definition of the Upper level sub-problem}

The upper level sub-problem is defined in a way that the solutions $\mathbf{x}, \mathbf{u}$ of the lower problem participate as parameters in its constraints and/or goal function. The upper level subproblem targets the maximization of the outflows from the arterial directions (left and right of Fig.1) by evaluating the duration of the time cycle of the traffic lights c. From practical and realistic requirements, the time cycle is constrained between upper $\mathbf{C}_{\max }$ and lower $\mathbf{C}_{\min }$ bounds.

The goal function is chosen in quadratic form to keep minimal cycle duration, which benefits the equilibrium distribution of waiting vehicles in overall network. The abnormal increase of the traffic cycles will generate congestion of the corresponding crossing sections.

Having the solutions of the lower level optimization problem $x$ and $u$, the upper level optimization problem becomes analytically defined. The arguments of the problem are the traffic cycle durations $c_{i}, i_{=} 1, \ldots, 5$, denoted below as vector $\mathbf{y}$ :

$$
\begin{aligned}
& \min _{\mathbf{y}} \mathbf{y}^{\mathbf{T}} \mathbf{y} \\
& \mathbf{C}_{\text {min }} \leq \mathbf{y} \leq \mathbf{C}_{\text {max }} \\
& u_{1}+u_{2}=0.9 y_{1} \\
& u_{3}+u_{4}=0.9 y_{2} \\
& u_{5}+u_{6}=0.9 y_{3} \\
& u_{7}+u_{8}=0.9 y_{4} \\
& u_{9}+u_{10}=0.9 y_{5}
\end{aligned}
$$


It is assumed the $10 \%$ of the traffic cycle $\mathbf{c}$ or $\mathbf{y}$ is used for the amber light. The resulting $90 \%$ of the cycle duration is used by the green lights in the both crossing directions.

The solutions of the upper level optimization problem give the duration of the cycles $\mathbf{c}=\left(c_{i}, i=1, \ldots, 5\right)$. These values are given to the lower-level problem where they participate as parameters for the definition of problem (10) for the new control cycle of the traffic lights.

\section{Numerical Simulation of the Bi-Level Control}

Problems (9) and (11) evaluate the duration of the green lights $\mathbf{u}$ and the cycles $\mathbf{c}$ for one control cycle. The sequentially solutions of these bi-level problems will give the dynamics of the control policy with the changes of the queue lengths and the outgoing flows. The numerical simulation uses as initial data the current estimates of the traffic behavior, which were performed for this urban network for peak time from 16:00-18:00 for three weeks. Comparisons have been done between the current applied plan of traffic lights and the numerically evaluations with the bi-level optimization.

The bi-level problem have been solved iteratively and on each iteration the obtained solutions of queue lengths, green light durations, and time cycles are used as input parameters for the next iteration. The numerical simulation was performed in MATLAB environment. Inside it has been install the bi-level toolbox YALMIP [32], which is a toolbox, implementing bi-level calculations. Particularly, the "solvebilevel" function was called in repeatedly way.

For the urban network it has been estimated values for the inflows as: $x_{1 \text { in }}=260 / 3600 ; \quad x_{2 \text { in }}=54 / 3600 ; \quad x_{3 \text { in }}=206 / 3600 ; \quad x_{6 i n}=$ $50 / 3600 ; x_{7 \text { in }}=90 / 3600 ; x_{10 \text { in }}=96 / 3600$

$x_{14 \text { in }}=270 / 3600 ; x_{17 \text { in }}=320 / 3600 ; x_{18 \text { in }}=250 / 3600$;

$x_{i o}=2, i=1, \ldots, 18$.

The current plan, applied for controlling the five intersections, has the following cycle durations

$c_{1}=60 ; \quad c_{2}=55 ; \quad c_{3}=55 ; \quad c_{4}=70 ; \quad c_{5}=60 ;$

$C_{\text {min }}=30 ; C_{\text {max }}=120$.

The green lights are fixed to values

$\mathbf{u}^{\mathbf{T}}=[27 ; 27 ; 25 ; 25 ; 25 ; 25 ; 32 ; 32 ; 27 ; 27]$.

The saturation flows are assessed according to the urban infrastructure with values

$\mathbf{s}^{\mathbf{T}}=[500 / 3600 ; 160 / 3600 ; 550 / 3600 ; 300 / 3600 ; 550 / 3600 ;$ 160/3600; 550/3600; 420/3600; 500/3600; 420/3600];

$\overline{\mathbf{s}}^{\mathbf{T}}=[200 / 3600 ; 160 / 3600 ; 157 / 3600 ; 148 / 3600 ; 250 / 3600 ;$ 150/3600; 490/3600; 240/3600; 240/3600; 20/3600].

The dynamical changes per time cycle of the 18 queue lengths $\mathbf{x}$ are presented graphically in Figures 4 till 22. The numerical simulations applied 10 control steps of the traffic network by solving the bi-level problem (9)-(11). It has been estimated that benefits of the total traffic behavior comes up to the 5-th step, because the majority of the vehicle queues vanish. That is the reason the illustrations of the results of the bi-level control in graphical mode to be up to 5-th control step. The horizontal axes of the figures below have meaning of sequence of the control steps.

A comparison between the queue lengths, resulting by bilevel control (in blue and solid line) and with the application of existing "fixed" time plans without optimization (red and dashed line) are presented in Figures 4 to 22.

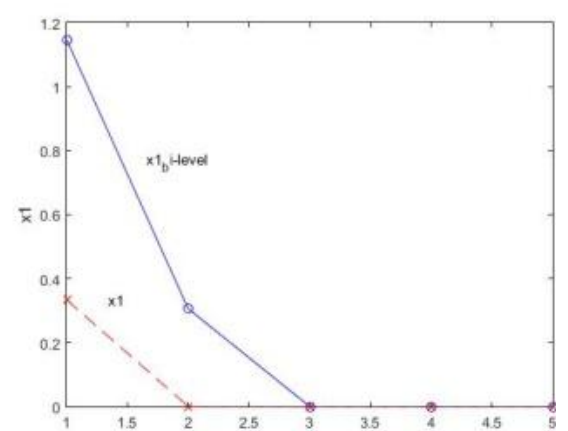

Fig.4 Queue $x_{1}$

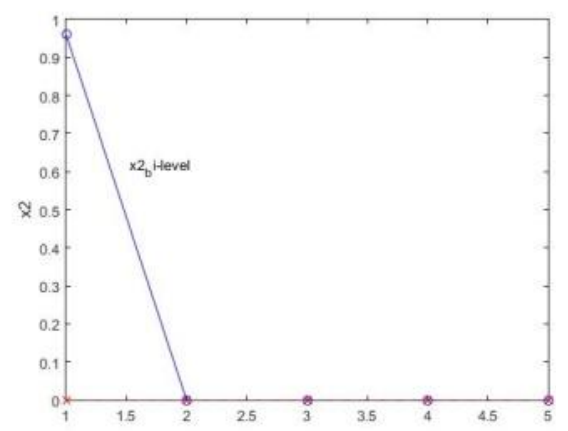

Fig.5 Queue $x_{2}$

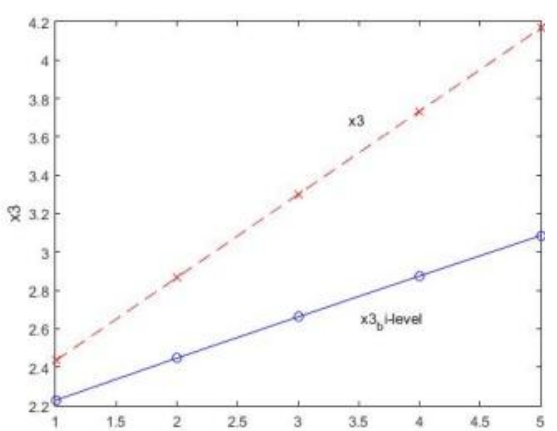

Fig.6 Queue $x_{3}$

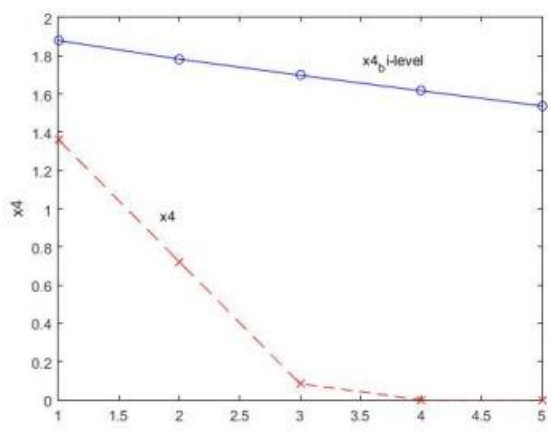

Fig.7 Queue $x_{4}$

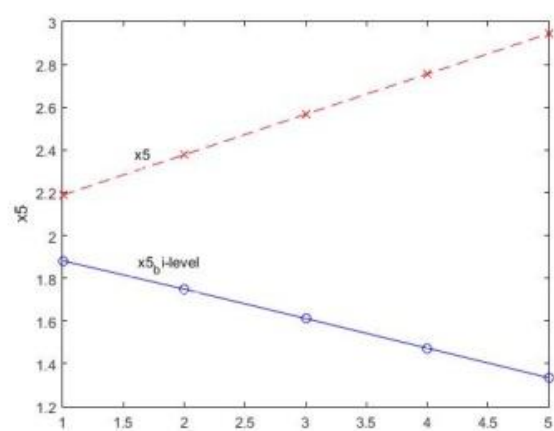

Fig.8 Queue $x_{5}$

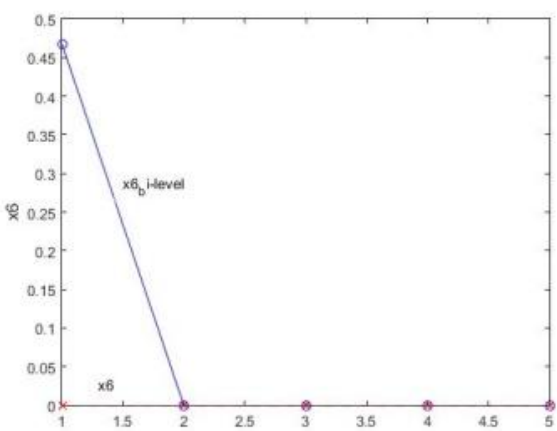

Fig.9 Queue $x_{6}$ 


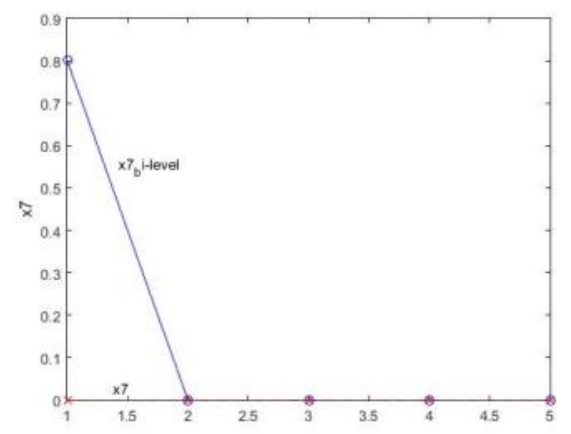

Fig.10 Queue $x_{7}$

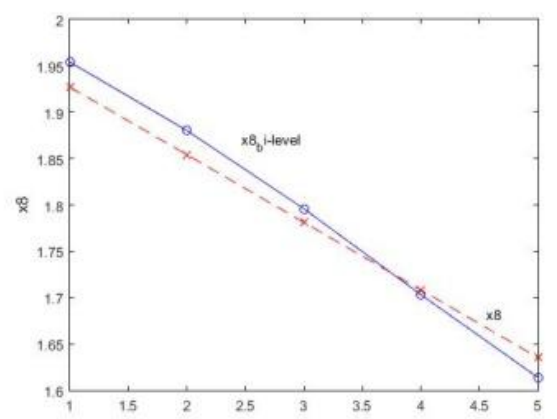

Fig.11 Queue $x_{8}$

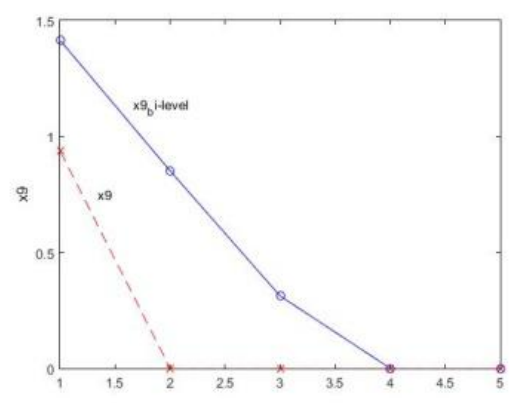

Fig.12 Queue $x_{9}$

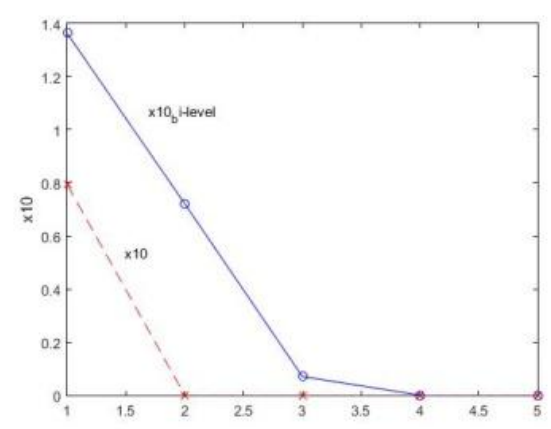

Fig.13 Queue $x_{10}$

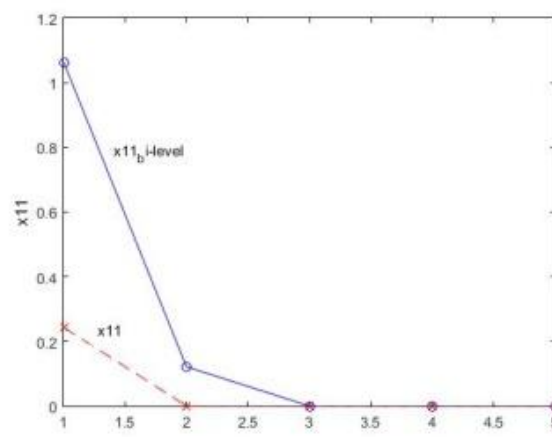

Fig.14 Queue $x_{11}$

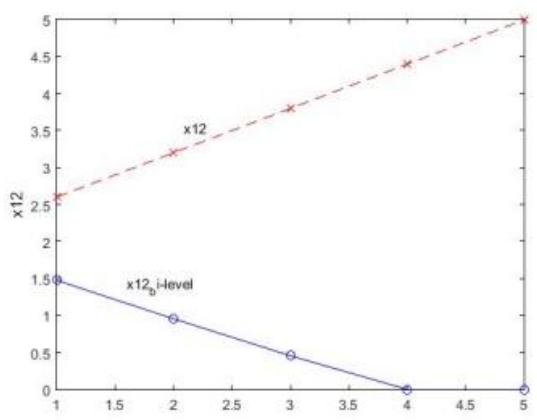

Fig.15 Queue $x_{12}$

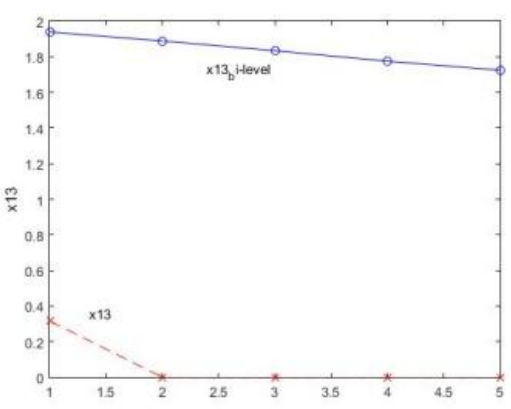

Fig.16 Queue $x_{13}$

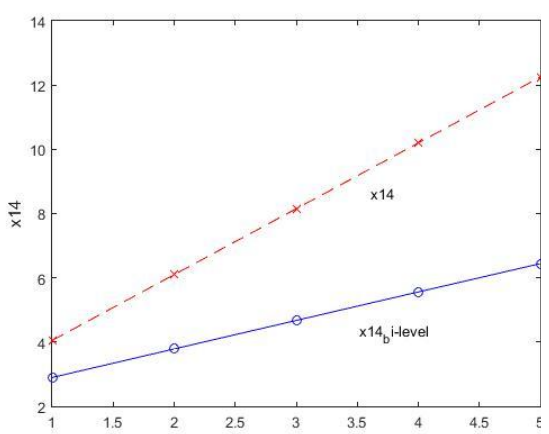

Fig.17 Queue $x_{14}$

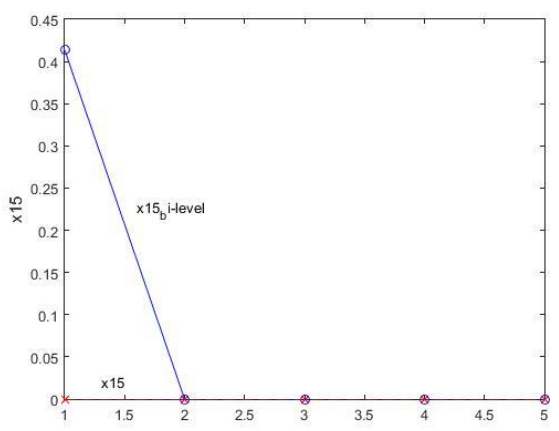

Fig.18 Queue $x_{15}$

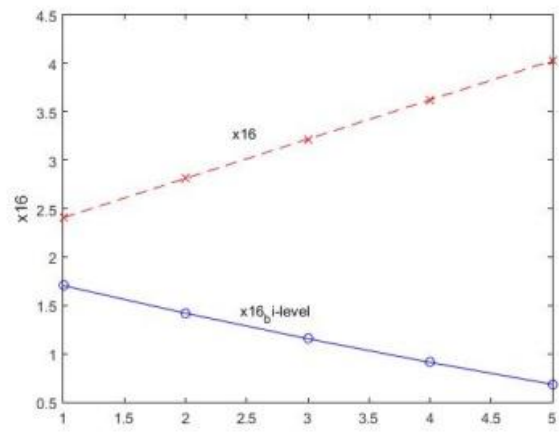

Fig.19 Queue $x_{16}$

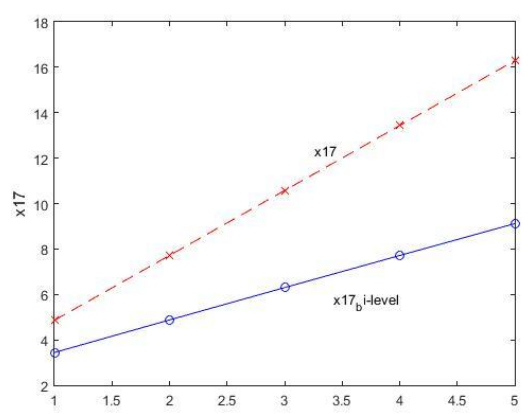

Fig.20 Queue $x_{17}$

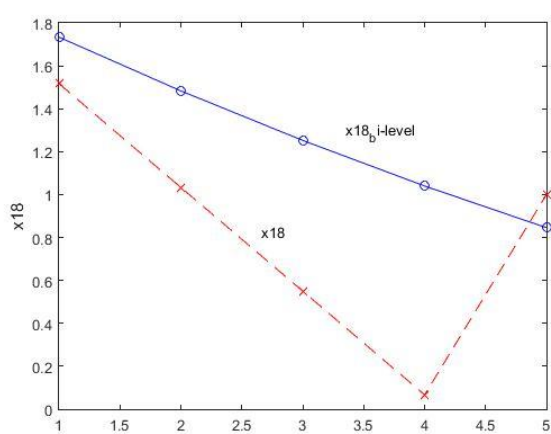

Fig.21 Queue $x_{18}$
The graphical assessment proves that the bi-level control optimization gives considerably better results in comparison with the existing "fixed" case of traffic control. All of the queue lengths keep decreasing behavior. It is obvious that the "fixed" control, currently applied on places, generates increasing character of the queue lengths, Figures 8, 9, 15 
and 21. The corresponding queue lengths $x_{5}, x_{12}, x_{16}$ and $x_{18}$ increase but with the application of the bi-level control the same queues have decreasing character. This is a benefit, which comes from this new bi-level formulation of the traffic control.

On Fig.22 it is presented an integral graphical comparison for the queue lengths for all queues with bi-level (blue solid lines) and the current "fixed" control (red dashed lines) strategies. The horizontal axis represents the traffic directions. It is evident that the "fixed" control strategy keeps higher levels of the queue lengths for the all 18 directions. The bi-level control maintains lower values of queues.

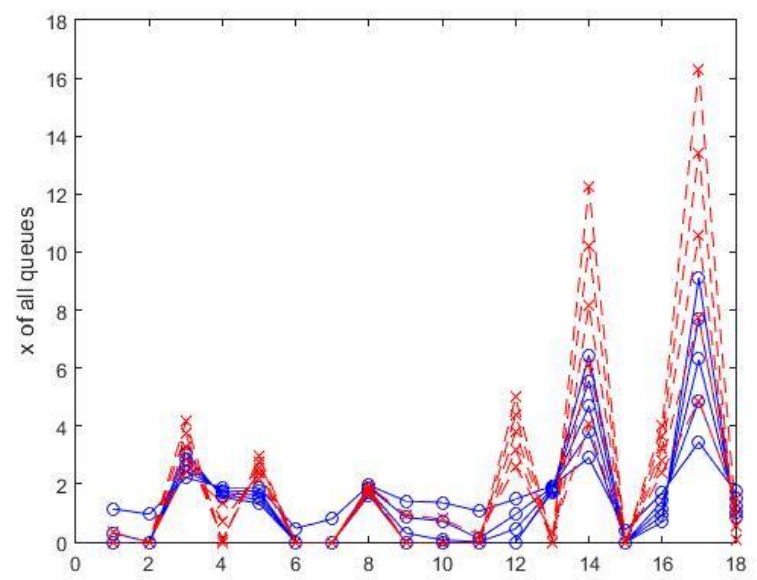

Fig.22 Comparison of all 18 queues $x_{i}$

Because the target of the bi-level control is to give preferences to the arterial directions (West to East and opposite) the next Figures 23 and 24 illustrate the case of the application of bi-level control. It is graphically proved that in both directions the queue lengths of the arterial directions have decreasing character and this prevents the occurrence of bottlenecks and congestions.

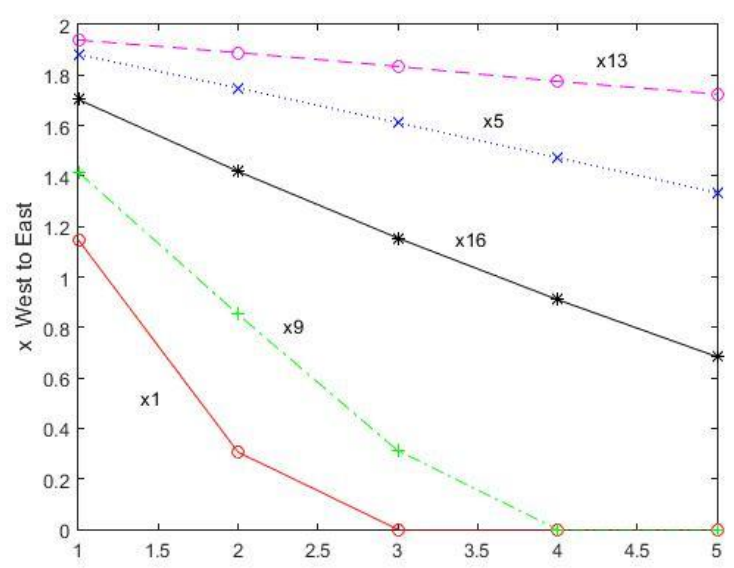

Fig.23 Queues $x_{i}$ from West to East
An integral comparison between the bi-level control strategy and the "fixed" time one is given in Fig.25. All vehicles, which are waiting in queues of this urban network are calculated as a sum of each individual queues. The bilevel control strategy gives total value of waiting vehicles in blue solid line, while the "fixed" time strategy is in red dashed line. It is evident that the "fixed" strategy has rapid increasing character, which explains the current situation of frequent congestions and bottlenecks on the arterial directions. In opposite, the bi-level policy decreases the total amount of waiting vehicles, which benefit the arterial outflows of the network.

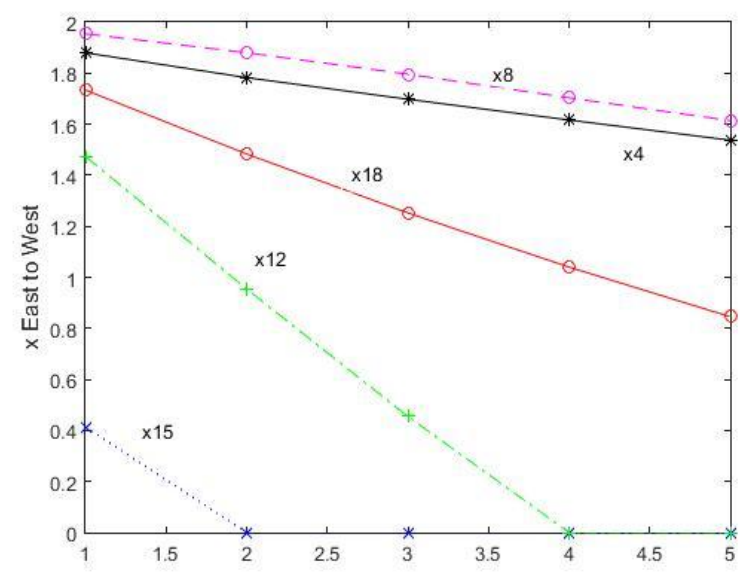

Fig.24 Queues $x_{i}$ from East to West

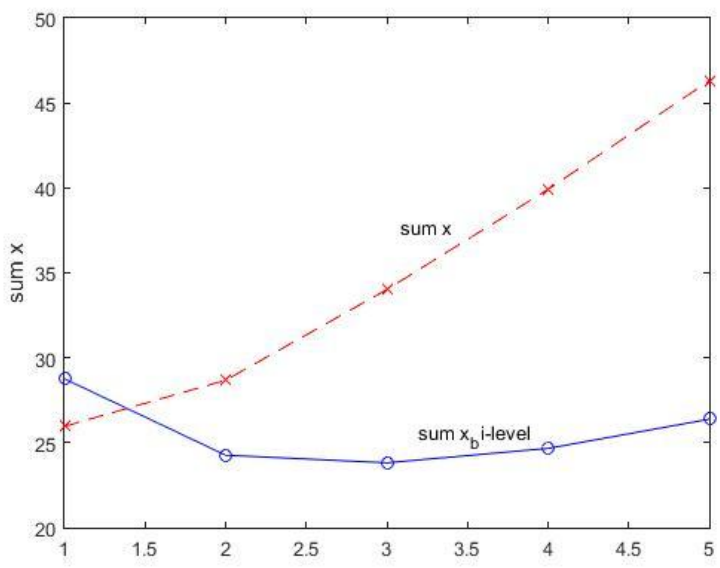

Fig.25 Comparison of the sum of all queues

For illustration purposes in Fig.26 are given the changes of the green lights durations, evaluated by the bi-level control. It is seen that they do not have constant values and the different crossroad sections apply different duration for the different control iterations. For the case of "fixed" time control the green lights per section are kept constant with values $u_{1}=27 ; \quad u_{2}=27 ; \quad u_{3}=25 ; u_{4}=25 ; \quad u_{6}=25 ; \quad u_{7}=25 ; u_{8}=32$; $u_{9}=32 ; u_{10}=27 ; u_{11}=27$. The bi-level control demonstrates sensibility and adaptive features for the evaluation of the green lights according to the current values of waiting vehicles. This is a benefit for the bi-level control. 


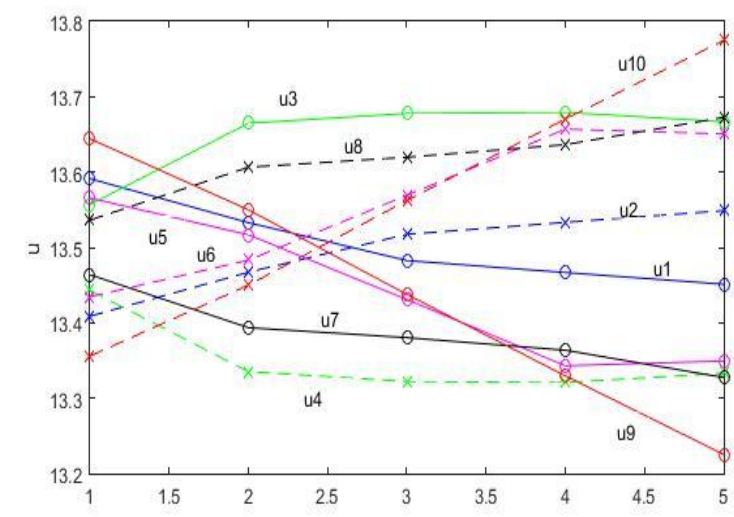

Fig.26 Optimization results of green light durations

\section{CONCLUSIONS}

An arterial transportation network with five crossroad sections is modeled in order to improve the traffic behavior by bi-level formalism. This hierarchical modeling allows extending the control space not only with the green light durations but with the duration of the control cycle of the traffic lights. The increased set of control influences results in better behavior of the traffic state: considerable reduction of the queue lengths in the total transportation network as to give priority in arterial direction. Thus, the bi-level optimal control allows reducing the appearance of events as bottlenecks and traffic congestions.

The bi-level modeling allows by integration of smaller optimization problems to have simultaneously two important control influences to the traffic: green lights ant time cycles. This research applies the store-and-forward modeling for the definition of the low optimization problem. The upper problem takes care of the outgoing flows by optimizing the cycle of the traffic flows. Thus, the application of the hierarchical approach benefits the control optimization procedure by optimizing simultaneously two important traffic control influences. The presented numerical simulation results illustrate the bigger potential and positive results of the bi-level control strategy. The comparisons with the currently established "fixed" plan control prove the benefit of the bi-level optimization control.

\section{REFERENCES}

[1] B. Park and J. D. Schneeberger, Evaluation of Traffic Signal Timing Optimization Methods Using a Stochastic and Microscopic Simulation Program, Virginia Transportation Research Council, 2003.

[2] W. H. Kraft, W. S. Homburger, and J. L. Pline, Traffic Engineering Handbook, Washington USA, Institute of Transportation Engineers, 2009.

[3] P. Koonce, L. Rodegerdts, K. Lee, S. Quayle, S. Beaird, C. Braud, J. Bonneson, P. Tarnoff, and T. Urbanik, Traffic Signal Timing Manual. Washington: Federal Highway Administration, 2008.

[4] K. Han, Y. Sun, H. Liu, T. L. Friesz, and T. Yao, "A bi-level model of dynamic traffic signal control with continuum approximation," Transportation Research Part C, vol.55, pp. 409-431, 2015, http://www.sciencedirect.com/science/article/pii/S0968090X1500126 6 , https://www.academia.edu/12549962/A_bi- level_model_of_dynamic_traffic_signal_control_with_continuum_ap proximation

[5] L. Li, D. Wen, and D. Yao, "A survey of traffic control with vehicular communications," IEEE Transactions on Intelligent Transportation Systems, vol.15, no 1, pp. 425-432, 2014,. DOI: 10.1109/TITS.2013.2277737

https://www.researchgate.net/publication/260720276_A_Survey_of_T raffic_Control_With_Vehicular_Communications

[6] R. P. Roess, E. S. Prassas, and W. R. McShane, Traffic Engineering, $5^{\text {th }}$ ed. Hoboken, NJ Pearson Education, 2019, ISBN-10:0-13-4599713 , ISBN-13:978-0-13-459971-7, https://www.pearsonhighered.com/assets/preface/0/1/3/4/0134599713. pdf

[7] H. Wei, G. Zheng, V. Gayah, and Z. Li, A survey on traffic signal control methods. Cornell University, 2020, https://arxiv.org/pdf/1904.08117.pdf,

[8] M. Papageorgiou, C. Diakaki, V. Dinopoulou, A. Kotsialos, and Y. Wang, Review of road traffic control strategies, in Proc. IEEE 91, 12, pp. 2043-2067, 2003.

[9] E. Eriskin, S. Karahancer, S. Terzi, and M. Saltan, Optimization of traffic signal timing at oversaturated intersections using elimination pairing system. 10th International Scientific Conference Transbaltica, Transportation Science and Technology, Procedia Engineering 187, pp. 295 - 300, 2017, doi: 10.1016/j.proeng.2017.04.378, https://www.sciencedirect.com/science/article/pii/S187770581731908 $\underline{2}$

[10] Y. Wang, X. Yang, H. Liang, and Y. Liu, "A Review of the SelfAdaptive Traffic Signal Control System Based on Future Traffic Environment," J. of Advanced Transportation, vol. 2018, Article ID 1096123, 12 pages, https://doi.org/10.1155/2018/1096123

[11] T. Tettamanti, I. Varga, and T. Peni, "MPC in urban traffic management," Model predictive control, Ed.T. Zheng, IntechOpen, 2010 DOI: 10.5772/9922. Available from: https://www.intechopen.com/books/model-predictive-control/mpc-inurban-traffic-management

[12] K. Aboudolas, M. Papageorgiou, and E. Kosmatopoulos, "Store-andforward based methods for the signal control problem in large-scale congested urban road networks," Transportation Research Part C, vol.1, pp. 163-174, 2009, doi:10.1016/j.trc.2008.10.002

[13] R. Scheffle and M. Strehler, "Optimizing Traffic Signal Settings for Public Transport Priority," 17th Workshop on Algorithmic Approaches for Transportation Modelling, Optimization, and Systems (ATMOS), 2017. G. D'Angelo and T. Dollevoet; Eds, Article No. 9; pp. 9:1-9:15, DOI:10.4230/OASIcs.ATMOS.2017.9

[14] V. Ivanov, "Monitoring of urban road transport,".Proc. of Intern conf Automatics and Informatics, 2017, pp. 135-141, ISSN:1313-1850.

[15] K. N. Hewage and J. Y. Ruwanpura, „Optimization of traffic signal light timing using simulation”, in Proc. 2004 Winter Simulation Conference, R. G. Ingalls, M. D. Rossetti, J. S. Smith, and B. A. Peters, Eds, 2004, pp.1428-1433, DOI: 10.1109/WSC.2004.1371482 .

[16] A. Jamal, M. T. Rahman, H. M. Al-Ahmadi, I. Ullah, and M. Zahid. Intelligent intersection control for delay optimization: using metaheuristic search algorithms. 2020, https://www.mdpi.com/20711050/12/5/1896/pdf

[17] L. N. Vicente and P. H. Calamai, "Bilevel and multilevel programming: A bibliography review," J Glob Optim, vol. 5, pp. 291306, 1994, https://doi.org/10.1007/BF01096458

[18] B. Colson, P. Marcotte, and G. Savard, "An overview of bilevel optimization," J. Ann Oper Res vol. 153, pp. 235-256, 2007, DOI 10.1007/s10479-007-0176-2, https://www.iro.umontreal.ca/ marcotte/ARTIPS/AOR2007.pdf

[19] S. A. Khandelwal and M. C. Puri, "Bilevel time minimizing transportation problem," J. Discrete Optimization, volume 5, no 4, pp. 714-723, $\quad$ November https://doi.org/10.1016/j.disopt.2008.04.004

[20] H. Sun, Z. Gao, and J. Wu, "A bi-level programming model and solution algorithm for the location of logistics distribution centers," $J$. Applied Mathematical Modelling, vol. 32, no 4, pp. 610-616, April 2008, https://doi.org/10.1016/j.apm.2007.02.007

[21] A. Arizti, A. Mauttone, and M. E. Urquhart, "A bilevel approach to frequency optimization in public transportation systems," in 18th Workshop on Algorithmic Approaches for Transportation Modelling, Optimization, and Systems (ATMOS 2018), .65, pp. 7:1-7:13, ISBN 978-3-95977-096-5, 
DOI:10.4230/OASIcs.ATMOS.2018.7,

http://drops.dagstuhl.de/opus/volltexte/2018/9712/

[22] J. Hao, X. Liu, X. Shen, and N. Feng, "Bilevel Programming Model of Urban Public Transport Network under Fairness Constraints," in Discrete Optimization for Dynamic Systems of Operations Management in Data-Driven Society, 2019, https://doi.org/10.1155/2019 /2930502,

[23] M. Patriksson, "Robust bi-level optimization models in transportation science," Philosophical transactions of royal Society A, vol. 366, no 1872, pp. 1931-1940, 2008, http://doi.org/10.1098/rsta.2008.0007

[24] R. Z. Farahania, E. Miandoabchib, W. Y. Szetoc, and H. Rashidid, "A review of urban transportation network design problems," European Journal of Operational Research, vol. 229, no 2, September 2013, Pages 281-302, doi: 10.1016/j.ejor.2013.01.001

[25] X. Jia, R. He, C. Zhang, and H. Chai, "A Bi-Level Programming Model of Liquefied Petroleum Gas Transportation Operation for Urban Road Network by Period-Security," Sustainability, MDPI, Open Access Journal, vol. 10, no 12, pp. 1-20, December 2018, https://ideas.repec.org/a/gam/jsusta/v10y2018i12p4714-d189583.html

[26] K. Moad, J. François , J. P. Bourrières , L. Lebel, and M. Vuillermo, "A bi-level decision model for timber transport planning", $6^{\text {th }}$ Int conf Information systems, logistics and supply chain, 2016 Bordeaux, http://ils2016conference.com/wp-content/uploads/2015/03/ILS2016_T D02_3.pdf
[27] C. Tawfik, S. Limbourg, "Bilevel optimization in the context of intermodal pricing: state of art," Transportation Research Procedia, vol. 10, pp. $634-643,2015$, https://orbi.uliege.be/bitstream/2268 /185274/1/1-s2.0-S2352146515002045-main.pdf, 10.1016/j.trpro .2015.09.017

[28] A. Sinha, P. Malo, and K. Deb, Transportation Policy Formulation as a Multi-objective Bilevel Optimization Problem, 2015, https://www.egr.msu.edu/ kdeb/papers/c2015009.pdf

[29] C. Lu, S. Yan, H. Ko and H. Chen, "A bilevel model with a solution algorithm for locating weigh-in-motion stations," in IEEE Transactions on Intelligent Transportation Systems, vol. 19, no. 2, pp. 380-389, Feb. 2018, https://ieeexplore.ieee.org/document/7922613

[30] R. G. Ródenas, M. L. L.García, M. T. S. Rico, and J. A. L. Gómez, "A bilevel approach to enhance prefixed traffic signal optimization," $J$. Engineering Applications of Artificial Intelligence, vol. 84, pp. 51-65, September 2019, https://doi.org/10.1016/j.engappai.2019.05.017

[31] S. Goel, S. F. Bush, and C. Gershenson, Self-Organization in Traffic Lights: Evolution of Signal Control w ith Advances in Sensors and Communications, June 2017, https://www.researchgate.net/ publication/319271996_Self-

Organization_in_Traffic_Lights_Evolution_of_Signal_Control_with Advances in Sensors and Communications

[32] https://yalmip.github.io/ 